ఠ

\title{
Amiodarone for the treatment and prevention of ventricular fibrillation and ventricular tachycardia
}

\author{
This article was published in the following Dove Press journal: \\ Vascular Health and Risk Management \\ 19 June 2010 \\ Number of times this article has been viewed
}

\author{
Hugo Van Herendael \\ Paul Dorian \\ Division of Cardiology, St. Michael's \\ Hospital, University of Toronto, \\ Toronto, Canada
}

\begin{abstract}
Amiodarone has emerged as the leading antiarrhythmic therapy for termination and prevention of ventricular arrhythmia in different clinical settings because of its proven efficacy and safety. In patients with shock refractory out-of-hospital cardiac arrest and hemodynamically destabilizing ventricular arrhythmia, amiodarone is the most effective drug available to assist in resuscitation. Although the superiority of the transvenous implantable cardioverter defibrillator (ICD) over amiodarone has been well established in the preventive treatment of patients at high risk of life-threatening ventricular arrhythmias, amiodarone (if used with a beta-blocker) is the most effective antiarrhythmic drug to prevent ICD shocks and treat electrical storm. Both the pharmacokinetics and the electrophysiologic profile of amiodarone are complex, and its optimal and safe use requires careful patient surveillance with respect to potential adverse effects.

Keywords: amiodarone, ventricular fibrillation, unstable ventricular tachycardia
\end{abstract}

\section{Introduction}

Amiodarone was developed originally as an antianginal agent in Belgium (Labaz Inc.) in $1962 .{ }^{1}$ Subsequently its antiarrhythmic abilities were observed; ${ }^{2}$ early investigations were primarily confined to Europe and South America where its use as an antianginal and antiarrhythmic gained widespread acceptance. ${ }^{3,4}$ The oral preparation (200 mg/tablet) was approved by the Food and Drug Administration (FDA) for use in the USA in 1985, and is indicated for adults with life-threatening ventricular arrhythmias when other treatments are ineffective or have not been tolerated. Intravenous amiodarone was approved by the FDA in 1995 for the same indication.

Amiodarone is used to manage virtually all forms of supraventricular and ventricular tachycardia and has therefore become one of the most frequently used antiarrhythmic drugs in clinical practice. ${ }^{5}$ This review will focus on the role of both the oral and intravenous preparation in the treatment and prevention of ventricular tachyarrhythmia.

\section{Pharmacology Pharmacokinetics}

The pharmacokinetics of amiodarone and its metabolites are complex. A basic understanding of the pharmacokinetics is important for the clinician to understand the antiarrhythmic properties of both the oral and intravenous preparation.

Amiodarone has a variable $(20 \%-80 \%)$ oral bioavailability. ${ }^{6,7}$ After absorption, the drug undergoes extensive enterohepatic circulation. A large first pass effect (hepatic P450 cytochrome oxidase-dependent oxidative de-ethylation) results in
Correspondence: Paul Dorian St. Michael's Hospital, Division of Cardiology, 30 Bond Street, 6-050 Q,Toronto, Ontario M5B IW8 Canada Tel +l 4I6-864-5I04

Fax + | 416-864-5849

Email dorianp@smh.toronto.on.ca 
mono-N-desethyl amiodarone (desethylamiodarone), which is active and has similar electrophysiologic effects as the parent compound. ${ }^{8}$ Peak amiodarone serum levels, after oral dosing, are achieved within 3-7 hours. The drug can then go through many other metabolic pathways to finally undergo glucuronidation, which precedes biliary clearance. Renal clearance is negligible, thus the dose of the drug does not have to be reduced in patients with renal failure, including dialysis-dependent patients.

Amiodarone (and desethylamiodarone) is highly lipophilic. ${ }^{9}$ This leads to a large volume of distribution and variable uptake into different tissues. A three-compartment model best explains the drug's kinetics. After absorption, serum levels in the central or plasma compartment initially increase rapidly. After the initial distribution phase, highest levels of amiodarone and desethylamiodarone are found in the deep compartment, which consists of adipose tissue, lung, liver and lymph nodes. Lowest levels are found in the peripheral compartment, which is composed of brain, muscles and thyroid. Drug levels in the peripheral and deep compartments rise at a much lower rate because of the large volumes of distribution, accounting for the long loading period. This model also explains the different phases of elimination of amiodarone. After administration of a single dose of amiodarone, a wide interpatient variable initial half-life is found. This plasma half-life ( $\alpha$ phase $t_{1 / 2 \alpha}$ ) represents the distribution into tissue compartments in which amiodarone is sequestered. Elimination from the peripheral and deep compartments (terminal elimination) is much slower and happens with a linear relationship between the amount of drug administered and the steady-state plasma level. ${ }^{7}$ After withdrawal of chronic oral amiodarone therapy, terminal elimination half-life $\left(\mathrm{t}_{1 / 2 \beta}\right)$ can be up to 60 days.

Data on the clinical usefulness of plasma concentrations have been conflicting. ${ }^{8}$ Some studies have suggested no correlation between the antiarrhythmic effect of amiodarone and plasma concentrations. Others have shown that plasma levels of $1.0-1.5 \mathrm{mg} / \mathrm{L}$ are associated with a decrease in the amount of ventricular ectopy, and that levels $>2.5 \mathrm{mg} / \mathrm{L}$ do not provide any additional antiarrhythmic benefit. In addition, some data suggest that plasma levels $>2.5 \mathrm{mg} / \mathrm{L}$ are associated with increased neurologic adverse reactions, and that higher desethylamiodarone to amiodarone concentration ratios $(>1.4)$ are associated with increased risk of toxicity. ${ }^{10}$

\section{Electrophysiology}

The electrophysiologic effects of amiodarone are very complex, still incompletely understood and unlike any other antiarrhythmic drug. There are important differences between the acute and chronic effects of amiodarone on cardiac tissue.

Acute amiodarone therapy results in a use-dependent inhibition of inward sodium (Vaughan-Williams class I effect) and inward calcium currents (Vaughan-Williams class IV effect), ${ }^{11}$ as well as a non-competitive alpha- and beta-blockade effect (Vaughan-Williams class II effect). ${ }^{12}$ Acute amiodarone therapy has no consistent effects on the repolarization phase of action potentials. ${ }^{11}$ Clinically these effects result in a depressed automaticity of the sinoatrial node (SA node) resulting in a decrease in sinus rate, conduction slowing and increased refractoriness of the atrioventricular (AV) node resulting in depression of AV node function, and a stabilizing effect during acute electrical instability. The QTinterval has been shown to be relatively unaffected by acute amiodarone and a prolongation of effective refractory period (ERP) in ventricular muscle is minimal or negligible. ${ }^{11,13}$

The major effect of chronic amiodarone therapy is an inhibition of outward potassium currents (Vaughan-Williams class III effect) resulting in a prolongation of action potential duration (APD), not only in atrial and ventricular muscles but also in the SA and AV nodes. This APD prolongation is associated with a comparable prolongation of the ERP. ${ }^{11}$ Unlike drugs with "pure" class III effect (eg, d-sotalol, dofetilide), the effect of amiodarone on APD and ERP is not reverse-use dependent, and the effect is preserved at high heart rates. ${ }^{14}$

\section{Efficacy}

Amiodarone has been shown to be effective for both the termination of ongoing ventricular arrhythmia, as well as for the prevention of recurrence of ventricular tachycardia/ ventricular fibrillation (VT/VF) during electrical storm or after the arrhythmia has subsided.

\section{During VT/VF}

The efficacy of amiodarone in the termination of ventricular arrhythmias has been tested in different clinical settings.

Two randomized clinical trials have evaluated IV amiodarone in out-of-hospital cardiac arrest. The Amiodarone in Out-of-Hospital Resuscitation of Refractory Sustained Ventricular Tachycardia (ARREST) study compared IV amiodarone $(300 \mathrm{mg}$ ) to placebo in a blinded, randomized trial in patients with shock-refractory out-of-hospital VF or pulseless VT; 44\% of amiodarone-treated patients and 34\% of placebo-treated patients survived to hospital admission $(P=0.03) .{ }^{15}$ The Amiodarone versus Lidocaine in Prehospital Ventricular Fibrillation Evaluation (ALIVE) study compared 
amiodarone $(5 \mathrm{mg} / \mathrm{kg})$ to lidocaine $(1.5 \mathrm{mg} / \mathrm{kg})$ in a blinded, randomized trial in patients with out-of-hospital VF, resistant to three shocks, intravenous epinephrine and a further shock, or recurrent VF after initial successful defibrillation; $22.8 \%$ of amiodarone-treated patients and $12 \%$ of lidocainetreated patients survived to hospital admission $(P=0.009) .{ }^{16}$ Unadjusted analysis of the latter trial found that, among patients whose initial rhythm was VF, the interval from the first shock to the administration of the drug was a significant predictor of survival (odds ratio [OR] for survival for each minute of delay, $0.87 ; 95 \%$ confidence interval $[\mathrm{Cl}]$ : 0.80 to $0.96 ; P=0.003$ ).

No placebo-controlled trials have addressed the effectiveness of amiodarone in terminating hemodynamically tolerated monomorphic ventricular tachycardia (mVT). The available case series suggest a low termination rate of $\mathrm{mVT}$ of $29 \%$ (95\% CI: 0.13 to 0.49$)^{17}$ and $29 \%$ (95\% CI: 0.18 to 0.45$)^{18}$ to $42 \%(95 \% \mathrm{CI}: 0.20 \text { to } 0.67)^{19}$ after administration of $150 \mathrm{mg}$ and $300 \mathrm{mg}$ IV amiodarone, respectively. All included patients had structural heart disease with moderately impaired left ventricular ejection fraction (LVEF), mainly secondary to ischemic heart disease. In comparison, one randomized, unblinded study comparing the efficacy of procainamide and lidocaine in terminating $\mathrm{mVT}$ in the absence of severe congestive heart failure or acute myocardial infarction, documented a termination rate of $79 \%$ and $19 \%(P<0.001)$, respectively. ${ }^{20}$

The Intravenous Amiodarone Multicenter Investigators Group evaluated the effect of intravenous amiodarone in hemodynamically destabilizing ventricular arrhythmia refractory to lidocaine, procainamide and bretylium in hospitalized patients with structural heart disease and absence of QTprolongation $(>0.5 \mathrm{~s})$ or evidence of drug-provocation. Two dose-ranging studies were conducted, randomizing patients to different dose regimens: 125,500 or $1000 \mathrm{mg}$ amiodarone $\mathrm{IV} / 24 \mathrm{~h}^{21}$ and 500,1000 or $2000 \mathrm{mg}$ amiodarone IV/24 h, ${ }^{22}$ respectively. Supplemental infusions $(150 \mathrm{mg})$ of intravenous amiodarone could be given to treat breakthrough ventricular arrhythmias. This was allowed to prevent premature study termination in the low dose groups, but potentially also obscured a differential drug effect at the higher doses with respect to recurrent VT/VF event rate (primary endpoint). A statistically significant dose-related increase was found in the time to first recurrence of arrhythmia between the $125 \mathrm{mg}$ and $1000 \mathrm{mg}$ dose group in the first study (median time to first event 9.8 hours and 13.7 hours, respectively; $P=0.030$ ) and between the $500 \mathrm{mg}$ and the combined $1000 \mathrm{mg}$ and $2000 \mathrm{mg}$ dose groups over the first 12 hours in the second study (4.8 hours and $>12$ hours, respectively; $P=0.046$ ). In both studies the mean number of supplemental infusions decreased significantly with increasing blinded amiodarone dose, suggestive for a higher efficacy of the higher doses. The frequency of amiodarone-related adverse effects was similar across all dose groups in both studies.

In a third study by the same group, patients were randomized to $125 \mathrm{mg}$ or $1000 \mathrm{mg}$ amiodarone IV/24 h or bretylium $2500 \mathrm{mg} / 24 \mathrm{~h}^{23}$ In this study, high-dose amiodarone was more effective than bretylium in preventing arrhythmia recurrence $(P=0.087)$, during hours 0 to 6 , when the largest number of patients were on blinded therapy. A higher incidence of hypotension was seen in the bretylium group. ${ }^{23}$

The pattern of VT/VF recurrences suggests that it requires 12-24 hours for IV amiodarone to reach full efficacy. As a result, it seems prudent to expect occasional recurrences early after amiodarone is administered; on the other hand, recurrences in the first 12-24 hours do not necessarily imply that the drug is destined to be ineffective. Due to its sodiumchannel blocking effects, amiodarone also significantly slows the rate of VT from its original rate in case of recurrence.

In this latter study, a minority of patients had incessant VT (ie, recurrent despite attempted electrical cardioversion). Although variation was not significant among the groups, the median time from initiation of therapy to termination of incessant VT was as follows: bretylium, 6.98 hours $(n=9)$, low-dose amiodarone, 4.58 hours $(\mathrm{n}=13)$; and high dose amiodarone, 4.23 hours $(\mathrm{n}=12)(P=0.62)$. This finding may provide useful information for the treatment of implantable cardioverter defibrillator (ICD) patients with electrical storm.

Unmasking of the Brugada ECG pattern type 1 has been described after administration of both oral and intravenous amiodarone. ${ }^{24,25}$ Amiodarone should therefore presumably be avoided in patients with the Brugada syndrome.

\section{Prevention of VT/VF}

Randomized clinical trials have established the superiority of the transvenous ICD over antiarrhythmic drugs in the preventive treatment of patients at high risk of life-threatening ventricular arrhythmias, both in primary and secondary prevention. ${ }^{26-29}$ The ICD has therefore become the treatment of choice for patients at risk of these arrhythmias.

However, the efficacy of amiodarone in the prevention of sudden cardiac death had already been well established in the pre-ICD era. A meta-analysis of all randomized trials showed that amiodarone reduced total mortality by 10 to $19 \% .^{30}$ The risk reduction was similar in primary prevention after myocardial infarction or in patients with 
congestive heart failure (CHF), and in secondary prevention after cardiac arrest. ${ }^{30}$ In a pooled database from 2 similar randomized clinical trials (the European Amiodarone Myocardial Infarction Trial (EMIAT) and the Canadian Amiodarone Myocardial Infarction Trial (CAMIAT)), that evaluated use of amiodarone in primary prevention in patients recovering from myocardial infarction, cardiac death and arrhythmic death or resuscitated cardiac arrest, were significantly lower in patients receiving amiodarone, compared to placebo ( $P=0.05$ and 0.03 , respectively), if they were also receiving beta-blockers. ${ }^{31}$ There appeared to be no benefit of amiodarone over placebo in patients not receiving beta-blockers.

In contrast, the Sudden Cardiac Death in Heart Failure Trial (SCD-HeFT) showed no survival benefit of treatment with amiodarone over placebo in primary prevention in patients with NYHA class II or III CHF and left ventricular ejection fraction $(\mathrm{LVEF}) \leq 35 \%$, despite a high use of beta-blocker $(>70 \%)$ in both the placebo and the amiodarone group. ${ }^{28}$ The findings were similar for all causes of CHF. In the meta-analysis of the ICD secondary prevention trials, patients with $\mathrm{LVEF}>35 \%$ appeared to obtain little or no survival benefit from the ICD compared with amiodarone, whereas those with moderate to severe left ventricular dysfunction obtained a significant benefit from the ICD. ${ }^{26}$

In summary, there appears to be no role for amiodarone therapy without concomitant ICD in patients with a stable chronic low LVEF, both in primary and secondary prevention. In secondary prevention, therapy with amiodarone (if used with a beta-blocker) may be a reasonable alternative to ICD in selected patients with $\mathrm{LVEF}>35 \%$, at least in the short term.

In ICD recipients amiodarone and ablation therapy may play an important role in the reduction of device therapy.

The Optimal Pharmacological Therapy in Cardioverter Defibrillator Patients (OPTIC) study was a randomized trial that compared amiodarone $(200 \mathrm{mg})$ plus beta-blocker with sotalol alone (240 mg, adjusted for renal function) or beta-blocker alone (bisoprolol $10 \mathrm{mg}$ or equivalent) in 412 patients who had received a dual-chamber ICD for inducible or spontaneous ventricular tachycardia (VT) or ventricular fibrillation (VF) and $\mathrm{LVEF} \leq 40 \%$ or syncope. ${ }^{32}$ All ICDs were optimally programmed to avoid shocks (ATP up to a rate of 222 bpm; SVT discriminators enabled). During one year of follow-up, shocks (appropriate and inappropriate) occurred in 41 patients (38.5\%) assigned to beta-blocker alone, in $26(24.3 \%)$ assigned to sotalol alone, and in $12(10.3 \%)$ assigned to amiodarone plus beta-blocker. Amiodarone plus beta-blocker significantly reduced the risk of any shock compared with beta-blocker alone (HR 0.27 ; 95\% CI: 0.14 to $0.52 ; P<0.001$ ) and sotalol (HR $0.43 ; 95 \% \mathrm{CI}: 0.22$ to $0.85 ; P=0.02$ ). There was a trend for sotalol to reduce shocks compared with beta-blocker alone (HR 0.61; 95\% CI: 0.37 to 1.01 ; $P=0.055)$. The rates of study drug discontinuation at 1 year were $18.2 \%$ for amiodarone, $23.5 \%$ for sotalol, and $5.3 \%$ for beta-blocker alone.

No well-conducted randomized clinical trial has been published that compares prophylactic ablation with antiarrhythmic drugs for the reduction of device therapy in ICD recipients. Two randomized trials have compared ICD implant and prophylactic ablation to ICD implant alone in secondary prevention in patients with a history of myocardial infarction. The SMASH-VT trial enrolled patients with a history of VT or VF, not treated with antiarrhythmic drugs. ${ }^{33}$ In that trial, prophylactic substrate-based catheter ablation reduced ICD shocks from $31 \%$ to $9 \%$ over a mean follow-up of $22.5 \pm 5$ months $(P=0.003)$, and reduced VT from $33 \%$ to $12 \%$ $(P=0.007)$. Substantial ablation-related complications (pericardial effusion, exacerbation of congestive heart failure and deep venous thrombosis) occurred in 5\% of patients. The 30-day mortality rate was zero after ablation. The VTACH trial enrolled patients with a history of stable VT. ${ }^{34}$ In both the ablation and control groups, 35\% of patients were treated with amiodarone. In this trial, the number of appropriate ICD therapy events per patient and per year was also lower in the ablation group than in the control group (median 0.2 vs $3.0 ; P=0.013$ ). $3.8 \%$ of patients from the ablation group developed substantial ablation-related complications (two ablation procedures were terminated prematurely because of transient ischaemic ST-segment elevation in one patient and a transient cerebral ischaemic event in another). Again, no deaths occurred within 30 days after ablation.

Based on the current evidence, we would recommend treating all ICD recipients with structural heart disease with beta-blockers at the time of ICD implant. If the patient develops a recurrence of VT or VF with symptoms or shocks, addition of amiodarone, with a maintenance dose of $200 \mathrm{mg}$ once daily, to beta-blockers should be considered. Although the results of the ablation trials are promising, it is not clear whether outcomes of ablation would be similar for nonischemic patients or in less experienced centers. At this point, ablation is not recommended as a first line treatment. 


\section{Safety and tolerability}

Amiodarone has been reported to cause a variety of cardiac and extracardiac side effects, both in its oral and intravenous formulation (Table 1).

\section{Oral formulation}

Of the extracardiac adverse effects, pulmonary fibrosis is the most serious since it is potentially fatal. The risk of pulmonary toxicity, as well as hepatic and cutaneous changes, has been found to increase with high daily doses over an increased length of time $(>500 \mathrm{mg} / \mathrm{d}) .^{35,36} \mathrm{In}$ one study, a higher occurrence of neurological and gastrointestinal side effects was associated with amiodarone plasma levels $>2.5 \mathrm{mg} / \mathrm{L} .{ }^{37}$ Corneal microdeposits are found in $76 \%-100 \%$ of patients on chronic therapy, but they do not cause permanent eye damage. ${ }^{10}$ Only about $10 \%$ of patients experience visual disturbances in the form of halos when looking at bright lights at night. ${ }^{10}$

A meta-analysis of 4 placebo-controlled, randomized trials, involving 1465 patients exposed to a low daily dose of amiodarone (152-330 mg/day) for a minimum of 12 months, showed significantly higher odds than those on placebo $(P<0.05)$ for experiencing thyroid (OR 4.2, 95\% CI: 2.0 to 8.7 ), neurologic (OR 2.0, 95\% CI: 1.1 to 3.7), skin (OR $2.5,95 \%$ CI: 1.1 to 6.2$)$ ), or bradycardic (OR $2.2,95 \% \mathrm{CI}$ : 1.1 to 4.3 ) adverse effects. ${ }^{38} \mathrm{~A}$ trend toward increased odds of pulmonary toxicity was noted (OR $2.0,95 \% \mathrm{CI}: 0.9$ to 5.3), but this did not reach statistical significance $(P=0.07)$. The unadjusted total incidence of drug discontinuation was $22.9 \%$ in the amiodarone group and $15.4 \%$ in the placebo group at 1 year of follow-up.

Most adverse effects are reversible with a decrease of amiodarone maintenance dose or discontinuation of the drug. ${ }^{35-37}$

The cardiac adverse effects associated with the chronic use of amiodarone include sinus bradycardia, high degree

Table I Adverse effects during long-term amiodarone therapy

\begin{tabular}{ll}
\hline Extracardiac & Cardiac \\
\hline Hypothyroidism/hyperthyroidism & Sinus bradycardia \\
Skin: sun sensitivity, skin discoloration & High degree AV block \\
Central nervous system: tremors, ataxia, & Torsade de Pointes \\
nightmares, parasthesiae & \\
Visual disturbances & \\
Gastrointestinal: constipation, anorexia, nausea & \\
Abnormal liver function: abnormal & \\
ALT and AST levels & \\
Pulmonary fibrosis & \\
Reduced clearance of other drugs: eg, warfarin, \\
digoxin, simvistatin, metoprolol, diltiazem, flacainide, \\
procainamide
\end{tabular}

AV-block and, rarely, torsades de pointes (TdP), a potentially life-threatening proarrhythmia. Patients chronically treated with amiodarone are at risk of TdP because of the prolongation of repolarization and, therefore, prolongation of the QT interval. Among the class III antiarrhytmic drugs, amiodarone and azimilide are associated with a lower risk for $\operatorname{TdP}(<1 \%)$ than dofetilide and sotalol $(3 \%$ and $2.5 \%$, respectively). ${ }^{39}$ Several risk factors have been identified for the development of TdP, including female gender, baseline long QT, concomitant therapy with other QT-prolonging agents, hypokalemia and hypomagnesia, bradycardia and structural heart disease (including left ventricular hypertrophy). Since amiodarone is not renally cleared, renal insufficiency is not a risk factor for TdP in patients treated with amiodarone, contrary to patients treated with sotalol and dofetilide. ${ }^{39}$ Amiodarone has also been safely used in patients with a history of drug-mediated TdP, despite comparable prolongation of $\mathrm{QT}_{\mathrm{c}}{ }^{40}$

Amiodarone interacts with the metabolism of many other drugs, because of its metabolism through the cytochrome $\mathrm{P} 450$ isozyme family in the liver. This leads to a reduced clearance of drugs such as warfarin, digoxin, simvastatin, metoprolol, diltiazem, flecainide, procainamide. A $30 \%-50 \%$ potentiation of the effect of warfarin, ${ }^{37}$ a doubling in serum digoxin levels, ${ }^{41}$ and a dose-dependent risk of rhabdomyolysis with simvastatin doses exceeding $20 \mathrm{mg}$ have been described. ${ }^{42}$

\section{Intravenous formulation}

The predominant clinical adverse event associated with the intravenous administration of amiodarone is hypotension, caused by a negative inotropic effect and a decrease in systemic vascular resistance. ${ }^{43}$ This effect is mainly caused by the cosolvents, polysorbate- 80 and benzyl alcohol, used in the currently available form of intravenous amiodarone. To minimize these effects the drug is diluted in 5\% dextrose in water and slowly infused. In three controlled trials, hypotension was reported in $10 \%-30 \%$ of patients. ${ }^{44}$ It was observed more commonly with rapid rates of infusion, but no significant differences among the different dose groups were seen.

Depression of sinus node function and AV block can occur and should always be anticipated.

Thrombophlebitis has been reported in $>50 \%$ of patients after administration of intravenous amiodarone through a peripheral vein..$^{45}$ To avoid this side effect, administration through a central intravenous line is recommended.

Acute hepatitis has been reported after administration of intravenous amiodarone,${ }^{46}$ but organ toxicity is rare and 
only noted after prolonged administration of the intravenous preparation. ${ }^{44}$

\section{Alternatives Oral formulation}

Dronedarone is a derivative of amiodarone with a similar electropharmacologic profile, but without iodine to eliminate the iodine-related adverse reactions. ${ }^{47}$ The ATHENA (A placebo-controlled, double-blind, parallel arm Trial to assess the efficacy of dronedarone $400 \mathrm{mg}$ bid for the prevention of cardiovascular Hospitalization or death from any cause in patiENts with Atrial fibrillation/atrial flutter) study, showed fewer cardiovascular hospitalizations or deaths when treated with dronedarone, compared to placebo. ${ }^{47}$ On the other hand, the Antiarrhythmic Trial with Dronedarone in Moderate to Severe CHF Evaluating Morbidity Decrease (ANDROMEDA) had to be halted early because of increased mortality in the dronedarone group. ${ }^{48}$ In this trial, patients who were hospitalized with symptomatic heart failure and severe left ventricular systolic dysfunction were randomized to receive $400 \mathrm{mg}$ of dronedarone twice a day or placebo. Dronedarone has not been specifically tested for VT/VF.

\section{Intravenous formulation}

Several practical difficulties arise when the current formulation of intravenous amiodarone has to be administered in an emergency setting. With this formulation amiodarone must be aspirated from glass ampoules (due to adsorption on to plastics and rubber), then filtered and diluted before use. When agitated or aspirated too rapidly the drug may foam which may compromise proper dosing. The current formulation is also incompatible with electrolyte solutions other than dextrose in water. Furthermore, the cosolvents are presumably largely responsible for the adverse effect of hypotension. ${ }^{43}$

These practical considerations may cause a considerable delay in drug administration and prevent bolus push of the drug. Since rapid drug administration has been proven important, efforts have been focused on the development of alternative formulations of amiodarone, including an emulsion with tocopherol, ${ }^{49}$ a suspension of amiodarone in 0.1 $\mathrm{M}$ acetate buffer at $\mathrm{pH} 3.8,{ }^{50}$ a suspension of amiodarone in lactate buffer, ${ }^{51}$ amiodarone solubilized in methoxy poly (ethylene oxide)-block-poly(ester) micelles ${ }^{52}$ and amiodarone solubilized in sulfobutylether-7-beta-cyclodextrin $\left(\right.$ Captisol $\left.^{\circledR}\right){ }^{53}$ In December 2008 this latter formulation, branded Nexterone ${ }^{\circledR}$, was approved by the FDA, with the same label indications as the previously approved formulation of amiodarone. The diluent, Captisol ${ }^{\circledR}$, is an FDA approved excipient, which is hemodynamically and electrophysiologically inert, and well-tolerated with no known organ toxicity in humans. ${ }^{54}$ This formulation of amiodarone is compatible with ionic solutions besides dextrose in water, does not adsorb to plastics, and can be packaged in pre-filled syringes that may be administered as an intravenous push immediately after establishing IV access. Administration of captisol-enabled amiodarone as a rapid IV bolus $(150 \mathrm{mg})$ and as an infusion is bioequivalent to the approved formulation of amiodarone, with identical electrophysiologic effects after bolus dosing. Hypotensive effects have not been observed after $150 \mathrm{mg}$ bolus intravenous administration. ${ }^{53,55}$

\section{Summary}

Despite the well-understood toxicity of amiodarone, it remains the most effective and safe, in the short term, antiarrhythmic drug for ventricular arrhythmias. It can be considered as first line therapy in patients with hemodynamically significant ventricular tachycardia, particularly if recurrent. In patients with an ICD, it can be used as adjunctive therapy to prevent VT or VF recurrences, especially in patients with frequent events or those with electrical storm.

\section{Disclosures}

The authors report no conflicts of interest in this work.

\section{References}

1. Singh BN. Amiodarone: Historical development and pharmacologic profile. Am Heart J. 1983;106:788-797.

2. Van Schepdael J, Solvay H: Etude Clinique De L'Amiodarone Dans Les Troubles du Rhythme Cardiaque. Presse Med. 1970;78:1849.

3. Singh BN, Vaughan Williams EM. The effect of amiodarone, a new anti-anginal drug, on cardiac muscle. $B r J$ Pharmacol 1970;39(4):657-667.

4. Rosenbaum MB, Chiale PA, Halpern MS, et al. Clinical efficacy of amiodarone as an antiarrhythmic agent. Am J Cardiol 1976;38(7):934-944.

5. Connolly SJ. Evidence-Based Analysis of Amiodarone Efficacy and Safety. Circulation. 1999;100(19):2025-2034.

6. Andreasen F, Agerback H, Bjerregaard P, Gotzsche H. Pharmacokinetics of amiodarone after intravenous and oral administration. Eur J Clin Pharmacol. 1981;19(4):293-299.

7. HoIt DW, Tucker CT, Jackson PR, Storey CGA. Amiodarone pharmacokinetics. Am Heart J. 1983;106:840-847.

8. Freedman MD, Somberg JC. Pharmacology and pharmacokinetics of amiodarone. J Clin. Pharmacol. 1991;31:1061

9. Anastasiou-Nana M, Lewis G, Moulopoulos S. Plasma levels of amiodarone after intravenous and oral administration. J Clin Chem Clin Biochem. 1981;19:599-600. 
10. Pollak PT. Clinical organ toxicity of antiarrhythmic compounds: ocular and pulmonary manifestations. Am J Cardiol. 1999;84(9A): 37R-45R.

11. Kodama I, Kamiya K, Toyama J. Cellular electropharmacology of amiodarone. Cardiovasc Res. 1997;35(1):13-29.

12. Charlier R. Cardiac actions in the dog of a new antagonist of adrenergic excitation which does not produce competitive blockade of adrenoceptors. Br J Pharmacol. 1970;39:668-674.

13. Morady F, DiCarlo LA Jr, Krol RB, Baerman JM, de Buitleir M. Acute and chronic effects of amiodarone on ventricular refractoriness, intraventricular conduction and ventricular tachycardia induction. $\mathrm{J} \mathrm{Am}$ Coll Cardiol. 1986;7(1):148-57.

14. Sager PT, Uppal P, Follmer C, Antimisiaris M, Pruitt C, Singh BN Frequency-dependent electrophysiologic effects of amiodarone in humans. Circulation. 1993;88(3):1063-1071.

15. Kudenchuk PJ, Cobb LA, Copass MK, et al. Amiodarone for resuscitation after out-of-hospital cardiac arrest due to ventricular fibrillation. N Engl J Med. 1999;341(12):871-878.

16. Dorian P, Cass D, Shwartz B, et al; Amiodarone as compared with lidocaine for shock resistant ventricular fibrillation. $N$ Engl J Med. 2002;346(12):884-890.

17. Marill KA, deSouza IS, Nishijima DK, et al. Amiodarone is poorly effective for the acute termination of ventricular tachycardia. Ann Emerg Med. 2006;47(3):217-224.

18. Tomlinson DR, Cherian P, Betts TR, Bashir Y. Intravenous amiodarone for the pharmacological termination of haemodynamically-tolerated sustained ventricular tachycardia: is bolus dose amiodarone an appropriate first-line treatment? Emerg Med J. 2008;25(1):15-18.

19. Schutzenberger W, Leisch F, Kerschner K, Harringer W, Herbinger W. Clinical efficacy of intravenous amiodarone in the short-term treatment of recurrent sustained ventricular tachycardia and ventricular fibrillation. Br Heart J. 1989;62(5):367-371.

20. Gorgels AP, van den Dool A, Hofs A, Mulleneers R, Smeets JL, Vos MA, Wellens HJ. Comparison of procainamide and lidocaine in terminating sustained monomorphic ventricular tachycardia. Am J Cardiol. 1996;78(1):43-46.

21. Scheinman MM, Levine JH, Cannom DS, et al. Dose-ranging study of intravenous amiodarone in patients with life-threatening ventricular tachyarrhythmias. The Intravenous Amiodarone Multicenter Investigators Group. Circulation. 1995;92(11):3264-3272.

22. Levine JH, Massumi A, Scheinman MM, et al. Intravenous amiodarone for recurrent sustained hypotensive ventricular tachyarrhythmias. Intravenous Amiodarone Multicenter Trial Group. J Am Coll Cardiol. 1996;27(1):67-75.

23. Kowey PR, Levine JH, Herre JM, et al. Randomized, double-blind comparison of intravenous amiodarone and bretylium in the treatment of patients with recurrent, hemodynamically destabilizing ventricular tachycardia or fibrillation. The Intravenous Amiodarone Multicenter Investigators Group. Circulation. 1995;92(11):3255-3263

24. Nägele H, Behrens S, Castel A. Ventricular tachycardia and aggravation of Brugada ECG pattern in a patient with coronary artery disease and combined amiodarone and beta-blocker therapy. Clin Res Cardiol. 2008;97(1):56-60.

25. Paul G, Yusuf S, Sharma S. Unmasking of the Brugada syndrome phenotype during the acute phase of amiodarone infusion. Circulation. 2006;114(11):e489-491.

26. Connolly SJ, Hallstrom AP, Cappato R, et al. Meta-analysis of the implantable cardioverter defibrillator secondary prevention trial. Eur Heart J. 2000;21(24):2071-2078.

27. Bokhari F, Newman D, Greene M, et al. Long-term comparison of the implantable cardioverter defibrillator versus amiodarone: eleven-year follow-up of a subset of patients in the Canadian Implantable Defibrillator Study (CIDS). Circulation. 2004;110(2):112-116.

28. Bardy GH, Lee KL, Mark DB, et al. Amiodarone or an implantable cardioverter-defibrillator for congestive heart failure. $N$ Engl J Med. 2005;352(3):225-237.
29. Lee KL, Hafley G, Fisher JD, et al. Effect of implantable defibrillators on arrhythmic events and mortality in the Multicenter Unsustained Tachycardia Trial. Circulation. 2002;106(2);233-238.

30. Sim I, McDonald KM, Lavori PW, et al. Quantitative overview of randomized trials of amiodarone to prevent sudden cardiac death. Circulation. 1997;96(9):2823-2829.

31. Boutitie F, Boissel JP, Connolly SJ, et al. Amiodarone interaction with beta-blockers: analysis of the merged EMIAT (European Myocardial Infarct Amiodarone Trial) and CAMIAT (Canadian Amiodarone Myocardial Infarction Trial) databases. Circulation. 1999; 99(17):2268-2275.

32. Connolly SJ, Dorian P, Roberts RS, et al. Comparison of beta-blockers, amiodarone plus beta-blockers, or sotalol for prevention of shocks from implantable cardioverter defibrillators. JAMA. 2006;295(2): 165-171.

33. Reddy VY, Reynolds MR, Neuzil P, et al. Prophylactic catheter ablation for the prevention of defibrillator therapy. $N \mathrm{Engl} \mathrm{J} \mathrm{Med.}$ 2007;357:2657-2665.

34. Kuck K-H, Schaumann A, Eckardt L, et al. Catheter ablation of stable ventricular tachycardia before defibrillator implantation in patients with coronary heart disease (VTACH): a multicentre randomised controlled trial. Lancet. 2010;375:31-40.

35. Rakita U, Sobel SM, Mostow N, et al. Amiodarone pulmonary toxicity. Am Heart J. 1983;106(4):906-914.

36. Pollak PT, You YD. Monitoring of hepatic function during amiodarone therapy. Am J Cardiol. 2003;91(5):613-616.

37. Haffajee CI, Love IC, Canada AT, et al. Clinical pharmacokinetics and efficacy of amiodarone for refractory tachyarrythmias. Circulation. 1983:67(6):1347-1355.

38. Vorperian VR, Havighurst TC, Miller S, January CT. Adverse effects of low dose amiodarone: a meta-analysis. J Am Coll Cardiol. 1997;30(3):791-798.

39. Wolbrette DL. Risk of proarrhythmia with class III antiarrhythmic agents: sex-based differences and other issues. Am J Cardiol. 2003;91(6A):39D-44D.

40. Mattioni TA, Zheutlin TA, Sarmiento JJ, et al. Amiodarone in patients with previous drug-mediated torsade de pointes. Long-term safety and efficacy. Ann Intern Med. 1989;111(7):574-580.

41. Nademanee K, Kannan R, Hendrickson J, et al. Amiodarone-digoxin interaction: clinical significance, time course of development, potential pharmacokinetic mechanisms and therapeutic implications. J Am Coll Cardiol. 1984;4(1):111-116.

42. Borders-Hemphill V. Concurrent use of statins and amiodarone. Consult Pharm. 2009;24(5):372-379.

43. Cushing DJ, Kowey PR, Cooper WD, et al. PM101: A cyclodextrin-based intravenous formulation of amiodarone devoid of adverse hemodynamic effects. Eur J Pharmacol. 2009;607(1-3):167-172.

44. Kowey PR, Marinchak RA, Rials SJ, et al. Intravenous Amiodarone. J Am Coll Cardiol. 1997;29(6):1190-1198.

45. Antonelli D, Barzilay J. Acute thrombophlebitis following IV amiodarone administration. Chest. 1983;84(1);120b-120.

46. Tagliamonte E, Cice G, Ducceschi V, et al. Acute hepatitis following amiodarone administration. Minerva Cardioangiol. 1997; 45(9):451-456.

47. Hohnloser SH, Crijns HJ, van Eickels M, et al. Effect of dronedarone on cardiovascular events in atrial fibrillation. $N$ Engl J Med. 2009;360(7):668-678.

48. Kober L, Torp-Pedersen C, McMurray JJV, et al. Increased mortality after dronedarone therapy for severe heart failure. $N$ Engl J Med. 2008;358(25):2678-2687.

49. Kessler D, Palepu N, Tustian A, et al. A novel amiodarone microemulsion injectable formulation. AAPS. Pharm Sci. 2002;4:W4122 (Abstract).

50. Somberg JC, Bailin SJ, Haffajee CI, et al. Intravenous lidocaine versus intravenous amiodarone (in a new aqueous formulation) for incessant ventricular tachycardia. Am J Cardiol. 2002;90(8):853-859. 
51. Kipp JE, Doty MJ, Rebbeck CL, Eilert JY, inventors; Baxter International, assignee. Amiodarone-containing parenteral administration. United States patent US 6479541. 2002 November 12.

52. Elhasi S, Astaneh R, Lavasanifar A. Solubilization of an amphiphilic drug by poly(ethylene oxide)-block-poly(ester) micelles. Eur J Pharm Biopharm. 2007;65:406-413.
53. Cushing DJ, Adams MP, Cooper WD, et al. Bioequivalence of 2 intravenous amiodarone formulations in healthy participants. J Clin Pharmacol. 2009;49(4):407-415.

54. Stella VJ, He Q. Cyclodextrins. Toxicol Pathol. 2008;36(1):30-42.

55. Prism Pharmaceuticals NDA for Captisol-enabled Amiodarone NDA; 2008 January.

\section{Publish your work in this journal}

Vascular Health and Risk Management is an international, peerreviewed journal of therapeutics and risk management, focusing on concise rapid reporting of clinical studies on the processes involved in the maintenance of vascular health; the monitoring, prevention and treatment of vascular disease and its sequelae; and the involvement of metabolic disorders, particularly diabetes. This journal is indexed on PubMed Central and MedLine. The manuscript management system is completely online and includes a very quick and fair peer-review system, which is all easy to use. Visit http://www.dovepress.com/ testimonials.php to read real quotes from published authors.

Submit your manuscript here: http://www.dovepress.com/vascular-health-and-risk-management-journal 